\section{Myasthenia Gravis Exacerbation Following Second Dose of mRNA-1273 Vaccine} Urvi G. Desai, MBBS, MS, MD ${ }^{1}$

\author{
${ }^{1}$ Carolinas Neuromuscular MDA Care Center \\ Atrium Health Neurosciences Institute
}

\section{Introduction}

A wide spectrum of central and peripheral neurological manifestations associated with severe acute respiratory syndrome coronavirus 2 (SARS-CoV-2) have been reported in the literature. Neuromuscular complications of SARS-CoV-2 infection resulting in coronavirus disease 2019 (Covid-19) range from the emergence of new findings to the exacerbation of existing neuromuscular disorders like myasthenia gravis. [1,2] Interim analysis by a physicianreported registry, COVID-19 Associated Risks and Effects in Myasthenia Gravis (CARE-MG), demonstrated that, of reported myasthenia gravis patients with COVID-19, 40\% of patients relapsed or experienced crisis requiring rescue therapy and 24\% died due to COVID-19. [3] To the best of our knowledge, however, exacerbation of myasthenia gravis has not been reported following currently available COVID-19 vaccinations. Here we report a case of myasthenia gravis exacerbation requiring hospitalization and rescue therapy following a second dose of the mRNA1273 vaccination.

\section{Case Report}

A 69-year-old female with a history of Myasthenia Gravis (MG) [by abnormal electrophysiological studies of single fiber electromyography suggestive of a postsynaptic neuromuscular junction disorder, binding AchR Ab: 0.44 nmol/L level (Athena Diagnostics), Abnormal binding of MGT-30 epitope of Titin (Washington University lab) and significantly elevated Antistriational Ab at 1:15360)] was in her usual state of health. However, she developed an acute onset of shortness of breath and generalized weakness with difficulty ambulating following the second COVID-19 vaccination (Moderna). Later in the evening of receiving vaccination she felt fatigued but remained afebrile. However, she woke up the next day with respiratory distress, had difficulty raising from bed and was seen in the emergency department. On arrival, she was tachypneic with a respiratory rate in the 20 s and tachycardic with HR of 110. Her examination showed use of accessory muscles of respiration and moderate proximal weakness. She was only able to count to 6 on a single breath. Her pulmonary function tests showed negative inspiratory force at $-40 \mathrm{~cm}$ of H2O. Chest x-ray showed no acute processes. She denied any other recent medication dose adjustments other than withholding her Pyridostigmine 48 hours for an SFEMG the day before she received the vaccination, which she had already resumed 20 hours prior to the vaccination. She was treated with plasma exchange, did not require intubation and continued on choline esterase inhibitors. She improved in her respiratory status with NIF at -52 . She gained strength enough to ambulate and climb stairs with assistance. Prior to this episode, her myasthenia gravis was managed with IVIG and choline esterase inhibitors. She was not yet on any other immunosuppressant as she had been unable to tolerate Mycophenolate Mofetil and was reluctant to take Azathioprine. Her insurance had declined coverage for Eculizumab. Her initial presentation was shortness of breath at rest in early 2019 with an initial MG ADL (MG Activities of Living Scale) of 10 and the most recent ADL prior to hospitalization was 2 .

\section{Discussion}

Currently, three Coronavirus Disease 2019 (COVID-19) vaccines have been granted emergency use and marketing authorization by the US Food and Drug Administration (FDA) and the European Medicines Agency (EMA) to combat the COVID-19 pandemic. Our patient received SARS-CoV-2 mRNA-1273 (Moderna) which is a messenger RNA vaccine. This vaccine encodes a stabilized version of the SARS-CoV-2 full-length spike glycoprotein trimer called S-2P. It is modified to include two proline substitutions at the top of the central helix in this $\mathrm{S} 2$ subunit. The mRNA is encapsulated in lipid nanoparticles at a concentration of $0.5 \mathrm{mg}$ per milliliter and diluted with normal saline for target vaccine concentration. In a phase 3 placebo-controlled trial enrolling 30,420 volunteers, the mRNA-1273 vaccine showed $94.1 \%$ efficacy at preventing Covid-19 illness, including severe disease. Aside from transient local and systemic reactions, no safety concerns were identified during that study. [4] In a study focusing on the subpopulation of adult patients specifically stratified according to age (56 to 70 years or $\geq 71$ years), which was a dose-escalation, open-label trial of mRNA-1273, adverse events were predominantly mild or moderate in severity and most frequently included fatigue, chills, headache, myalgia, and pain at the injection site. Such adverse events were dose-dependent and were more common after the second immunization. [5] However, it should be noted that in both the Pfizer-BioNTech COVID-19 Vaccine and Moderna COVID-19 Vaccine clinical trials, incidents of Bell's palsy were cited as medically attended adverse events (MAAE). Because of that, considering the biological plausibility, FDA recommends surveillance for cases of Bell's palsy with the deployment of the Moderna COVID-19 Vaccine 


\section{Clinic Stuff}

into larger populations. [6] However, it is imperative that such observations are cautiously widened to include other neurological comorbidities. The temporal relationship of MG exacerbation in our patient strongly suggests that it was related to the mRNA-1273 vaccination. Given the surveillance importance of emerging data, we believe that this case should be shared with the scientific community in a timely fashion.

\section{References}

1. Guidon, A.C. and A.A. Amato, COVID-19 and neuromuscular disorders. Neurology, 2020. 94(22): p. 959-969. doi: 10.1212/WNL.0000000000009566

2. Paliwal, V.K., et al., Neuromuscular presentations in patients with COVID-19. Neurol Sci, 2020. 41(11): p. 3039-3056. doi: 10.1007/sl0072-020-04708-8

3. Muppidi, S., et al., COVID-19-associated risks and effects in myasthenia gravis (CARE MG). Lancet Neurol, 2020. 19(12): p. 970-971. doi: 10.1016/S14744422(20)30413-0

4. Baden, L.R., et al., Efficacy and Safety of the mRNA1273 SARS-CoV-2 Vaccine. N Engl J Med, 2021. 384(5): p. 403-416. doi: 10.1056/NEJMoa2035389
5. Anderson, E.J., et al., Safety and Immunogenicity of SARS-CoV-2 mRNA-1273 Vaccine in Older Adults. N Engl J Med, 2020. 383(25): p. 2427-2438. doi: 10.1056/ NEJMoa2028436

6. Repajic, M., et al., Bell's Palsy after second dose of Pfizer COVID-19 vaccination in a patient with history of recurrent Bell's palsy. Brain Behav Immun Health, 2021. 13: p. 100217. doi: 10.1016/j.bbih.2021.100217

\section{Supplemental References}

1. US Food and Drug Administration (2020) PfizerBioNTech COVID-19 vaccine emergency use authorization review memorandum. https://www.fda.gov/media/144416/ download. Accessed 15 Jan 2021

2. US Food and Drug Administration (2020) Moderna COVID-19 vaccine emergency use authorization review memorandum. $\quad$ https://www.fda.gov/media/144673/ download. Accessed 15 Jan 2021

3. US Food and Drug Administration (2021) Janssen COVID-19 Vaccine EUA Letter of Authorization (fda.gov) 